\title{
Leopard spot retinal pigmentation in infancy indicating a peroxisomal disorder
}

\author{
C J Lyons, G Castano, A Q McCormick, D Applegarth
}

Br J Ophthalmol 2004;88:191-192

Background: Neonatal adrenoleucodystrophy (NALD) is a rare disorder resulting from abnormal peroxisomal biogenesis. Affected patients present in infancy with developmental delay, hypotonia, and seizures. Blindness and nystagmus are prominent features. The authors suggest a characteristic leopard spot pigmentary pattern in the peripheral retina to be diagnostic.

Methods: Three patients are reported with this presentation; the characteristic retinal appearance resulted in early diagnosis for one of these.

Conclusion: Leopard spot retinopathy in an infant with hypotonia, seizures, developmental delay, with or without dysmorphic features and hearing impairment, is a clue to the diagnosis of NALD.

$\mathrm{N}$ eonatal adrenoleucodystrophy (NALD), a rare autosomal recessively inherited disorder was first reported in 1978 by Ulrich et al. ${ }^{1}$ Visual loss is an early and prominent feature, often starting in infancy. Other clinical features include seizures, hypotonia, developmental delay, and deafness. ${ }^{2}$ Hepatomegaly and liver dysfunction occur early in life and mild craniofacial abnormalities may be present. Although adrenal insufficiency is clinically rare, adrenal cortical atrophy is a universal necropsy finding. Most neurological findings are due to demyelination, which together with liver dysfunction usually cause death before the age of 7 years.

We report three cases of NALD with characteristic retinal findings.

\section{CASE REPORTS}

Patient 1 presented in 1979, aged 3 months, with focal seizures and developmental delay. On examination, he was hypotonic, had low set ears, hypertelorism, and liver enlargement. He was visually inattentive. Fundus examination revealed a striking peripheral leopard spot pigmentary retinopathy. Visual evoked potentials were grossly disorganised and electroretinogram (ERG) showed no detectable response. Multiple endocrine and metabolic tests, including plasma and CSF amino acids, were all normal. Lysosomal diseases were excluded. A liver biopsy showed an abnormally prominent smooth endoplasmic reticulum (SER) the significance of which was not clear at that stage (the first preliminary report of SER abnormalities in neonatal adrenoleucodystrophy had only been published in 1978). No reference was made to the peroxisomes (peroxisomal abnormalities were first identified in neonatal adrenoleucodystrophy in 1982). The patient died without a definite diagnosis at 5 years of age but necropsy revealed an enlarged cirrhotic liver, adrenal cortical atrophy, and white matter degeneration consistent with a diagnosis of neonatal adrenoleucodystrophy.
Patient 2 was born by spontaneous delivery at 34 weeks gestation. Mild dysmorphic features were noted including bilateral epicanthic folds and transverse palmar creases. Subtle clouding of the right cornea disappeared in the first week of life. A complete eye examination, including dilated indirect ophthalmoscopy, was otherwise normal. He was brought to the hospital at the age of 3 months with seizures. Clinical examination showed a hypotonic infant with poor head control, complete visual inattention and horizontal nystagmus, sluggish pupil reflexes, and peripheral leopard spot pigmentary retinopathy (fig 1). Hepatomegaly and hearing impairment were also evident.

Computed tomography (CT) brain scan showed mild ventricular dilatation. The electroencephalogram (EEG) confirmed a seizure disorder with a structural abnormality. The ERG and visual evoked potentials (VEP) were abnormal but not diagnostic. Brainstem auditory evoked responses were abnormal; pituitary and adrenal function were normal. Markedly elevated levels of very long chain fatty acids (VLCFA) were found in plasma and fibroblasts at the age of 8 months, and a liver biopsy showed absence of peroxisomes with micronodular cirrhosis. A diagnosis of neonatal adrenoleucodystrophy was made.

The patient developed progressive feeding problems and signs of portal hypertension. By the age of 4 years, he was blind with marked developmental delay and poor seizure control. Fundus examination showed extreme narrowing of the retinal blood vessels with bilateral optic atrophy. The retinal pigment epithelium was atrophic and the leopard spot pattern had disappeared. The patient and his family subsequently moved and were lost to follow up.

Patient 3 presented at 5 months of age with developmental delay and visual inattention. Ocular examination showed a visually inattentive boy with small amplitude horizontal nystagmus. A bilateral peripheral leopard spot pigmentary retinopathy was evident on funduscopy. Electroretinography

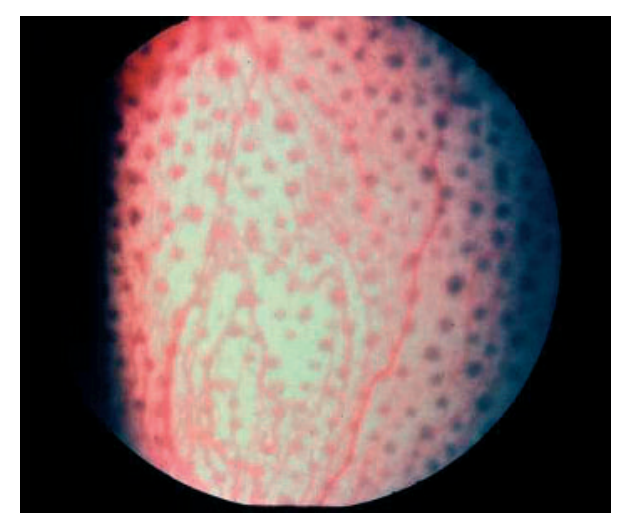

Figure 1 Fundus photograph of patient 1. The mid-peripheral retina shows a classic leopard spot pigmentary retinopathy. 
showed diffusely abnormal retinal function. CT brain scan was normal. In view of our experience with patients 1 and 2, the presence of bilateral retinal leopard spots in infancy suggested neonatal adrenoleucodystrophy. Plasma VLCFA levels were found to be elevated, in particular hexacosanoic acid (C26), and the C26:C22 ratio was elevated. Liver biopsy suggested an evolving cirrhosis and electron microscopy confirmed absence of the peroxisomes. The patient died at the age of 4 . Necropsy was refused.

\section{DISCUSSION}

The cause of neonatal adrenoleucodystrophy is the absence or near absence of peroxisomes, intracellular organelles exclusively responsible for the catabolism of long chain fatty acids ( 16 or more carbon atoms), very long chain fatty acids (22 or more carbon atoms), and pipecolic acid. ${ }^{3}{ }^{4}$ Peroxisomes are also important in plasmalogen and bile acid synthesis; their absence therefore results in increased levels of plasma VLCFAs, bile acid intermediates and pipecolic acid, and decreased red blood cell plasmalogen levels. These metabolic disturbances are particularly important in cells which are normally rich in peroxisomes such as hepatocytes, oligodendrocytes, and neurons in the first weeks of life. Tissue VLCFA accumulation causes the neurological and ocular findings; decreased plasmalogen levels may also affect the photoreceptor outer segment/retinal pigment epithelium relation since they are cell membrane constituents.

The most important ocular manifestations are optic atrophy and pigmentary retinopathy. Ganglion cell loss and gliosis of the nerve fibre layer are also found. ${ }^{5}$ Visual impairment results from these abnormalities as well as posterior visual pathway dysfunction. Because of the association of pigmentary retinopathy and deafness, NALD may be misdiagnosed as Usher's syndrome. However the retinopathy of NALD should be quite distinct from the appearance of retinitis pigmentosa, ${ }^{6}$ though a spicular pattern has been described in some cases and others were said to have "clumped and scattered" pigment. "Leopard spot" pigmentation was very characteristic in our three patients in whom small, round, discrete, evenly pigmented, and spaced areas were evident. Leopard spots have been described in other contexts including leukaemia, ${ }^{7}$ uveal effusion syndrome, ${ }^{8}$ systemic argyrosis, ${ }^{9}$ pseudoxanthoma elasticum, Warburg syndrome, ${ }^{10}$ and $\beta$ thalassaemia. These are clinically different from NALD. Leopard spot pigmentation appears to be a transient phenomenon in NALD, as in patient 2 whose abnormal pigmentation was absent at birth, evident at 3 months, and had disappeared by age 4 , when vascular narrowing and optic atrophy were the main funduscopic features.

Histopathologically, the pigmentary changes are associated with areas of photoreceptor degeneration ${ }^{11}$ with patchy hypertrophy, nodular hyperplasia, and atrophy of the pigment epithelium. Pigmented cells can be found in the subretinal space and in other layers in the retina. Macrophages may contain pigment and degenerated photoreceptors. A typical bileaflet inclusion is evident in the photoreceptor cells, pigmented epithelium, inner nuclear layer neurons, ganglion cells, and macrophages by electron microscopy. These inclusions can also be present in the brain, adrenal glands, and liver.

The ERG, VEP, and brain auditory evoked responses ${ }^{12}$ are almost always abnormal; these and plasma VLCFA assay should confirm this diagnosis. In atypical cases, other tests like plasma/urine bile acid intermediates, plasma pipecolic and phytanic acid, and peroxisomal enzymatic activity in cultured skin fibroblasts may be necessary. Peroxisome pathology may be evident on liver biopsy. A skin biopsy to establish cultured fibroblasts is required to confirm and investigate the absence or near absence of liver peroxisomes.

There is a clinical and biochemical overlap between NALD and Zellweger's (cerebro-hepato-renal) syndrome. ${ }^{13-15}$ Unlike $X$ linked childhood adrenoleucodystrophy, both these disorders have autosomal recessive inheritance. The presence of moderate to severe dysmorphic changes, renal cortical cysts and lack of adrenal cortical atrophy support a diagnosis of Zelleweger's syndrome rather than NALD. Also, severity of disease ranges from Zellweger syndrome, through NALD, to infantile Refsum disease in which the deficit of peroxisomes is least severe.

\section{CONCLUSION}

Leopard spot retinopathy has not been reported with NALD in the scientific literature. Although there is no treatment, early diagnosis avoids unnecessarily invasive, distressing, time consuming and expensive tests. This may help an affected family to come to terms with the prognostic implications and provide an opportunity for immediate genetic counselling.

\section{Authors' affiliations}

C J Lyons, G Castano, A Q McCormick, Department of Ophthalmology, British Columbia Children's Hospital, 4480 Oak Street, Vancouver BC, Canada V6H 3V4

D Applegarth, Department of Pediatrics, British Columbia Children's Hospital, 4480 Oak Street, Vancouver, BC, Canada V6H 3V4

Correspondence to: Christopher Lyons, Department of Ophthalmology, A136 BC Children's Hospital 4480 Oak Street, Vancouver, BC, Canada V6H3V4; cilyons@telus.net

Accepted for publication 4 April 2003

\section{REFERENCES}

1 Ulrich J, Herschkowitz N, Heitz P, et al. Adrenoleukodystrophy: preliminary report of a conatal case: light- and electron microscopical, immunohistochemical and biochemical findings. Acta Neuropathol (Berl) 1978:43:77-83.

2 Dimmick JE, Applegarth DA. Pathology of peroxisomal disorders. In: Landing BH, Haust MD, Bernstein J, Rosenberg HS, eds. Genetic metabolic diseases. Perspect Pediatr Pathol Basel Karger 1993;17:45-98.

3 Folz SJ, Trobe JD. The peroxisome and the eye. Surv Ophthalmol 1991;35:353-68

4 Wanders RJA, Schuutgens RBH, Tabak HJ. Functions and biogenesis of peroxisomes. In: Applegarth DA, Dimmick JE, Hall JG, eds. Organelle diseases. London: Chapman and Hall Medical 1997:147-67.

5 Wray S, Cogan DG, Kuwabara T, et al. Adrenoleukodystrophy with disease of the eye and optic nerve. Am J Ophthalmol 83:480-5.

6 Glasgow BJ, Brown HH, Hannah JB, et al. Ocular pathologic findings in neonatal adrenoleukodystrophy. Ophthalmology 1978;94:1054-60.

7 Clayman HM, Flynn JT, Koch K, et al. Retinal pigment epithelial abnormalities in leukemic disease. Am J Ophthalmol 1972;74:416-19.

8 Schepens CL, Brockhurst RJ. Uveal effusion 1: Clinical picture. Arch Ophthalmol 1963:70:189-201.

9 Cohen S, Quentel G, Egasse D, et al. The dark choroid in systemic argyrosis. Retina 1993;13:312-16.

10 Barth RA, Pagon RA, Bunt-Milam AH. Leopard spot retinopathy in Warburg syndrome. Ophthalmic Pediatr Genet 1986;7:91-6.

11 Cohen SM, Green WR, De La Cruz ZC, et al. Ocular histopathologic studies of neonatal and childhood adrenoleukodystrophy. Am J Ophthalmol 1983;95:82-96.

12 Verma NP, Hart ZH, Nigro M. Electrophysiologic studies in neonatal adrenoleukodystrophy. Electroencephalogr Clin Neurophysiol 1985;60:7-15.

13 Moser H, Moser AE, Singh I, O'Neill BP. Annals Neurol 1984;16:628-41.

14 Brown FR, Mc Adams AJ, Cummins JW, et al. Cerebro-hepato-renal (Zellweger) syndrome and neonatal adrenoleukodystrophy: similarities in phenotype and accumulation of very long chain fatty acids. Johns Hopkins Med J 1982;151:344-61

15 Cohen SM, Brown FR, Martyn L, et al. Ocular histopathologic and biochemical studies of the cerebrohepatorenal syndrome and its relationship to neonatal adrenoleukodystrophy. Am J Ophthalmol 1983;96:488-501. 